\title{
Food analysis and Foodomics
}

\author{
Alejandro Cifuentes
}

https://doi.org/10.1016/j.chroma.2009.09.018

For years, assessment of food quality and authenticity has been of great importance to guarantee the safety of food in compliance with legislation. This includes the control of technological processes and their effect on food, characterization of food composition, determination of food nutritional value, etc. These classical issues have been (and still are) of key importance for research institutions, industries, agencies and regulatory laboratories working in Food Science and Technology. Besides, detection and characterization of (new) food ingredients with demonstrated beneficial effects on human health is a major topic in modern Food Science, as demonstrated by the growing activity in the very new field of Foodomics that we have defined as a discipline that studies the Food and Nutrition domains through the application of omics technologies. In this context, Nutrigenomics and Nutrigenetics can be considered a part of the more general Foodomics term.

The complex mixture of aims mentioned above can only be addressed thanks to the large number of advanced analytical technologies available today, being the present special issue on "Food Analysis" a good example of this. Namely, this current issue is composed of 3 review articles plus 24 research papers. In the review articles, the use of modern multidimensional chromatographic techniques in food analysis, the use of mass spectrometry-based techniques to analyse food proteins and peptides, and the use of new separation methods to study food anthocyanins, isoflavones and flavanols are reviewed. Regarding the research papers, they are a good indication of the current and general trend towards using hyphenated techniques, with good examples on the use of LC-MS, GCMS and CE-MS in food analysis. Besides, the use of multidimensional chromatographic techniques is well represented, together with interesting applications of nano-liquid chromatography and capillary electromigration techniques. New developments in food sample preparation are also shown including the application of ionic liquidsbased microextracion, carbon nanotubes-based extraction or the use of pressurized fluids for analytes extraction. Also, a new method for the detection of radical scavengers in non-polar food matrices is presented. Last, but no least, some new Foodomics approaches are shown, including a comparative metabolomics study of transgenic vs. wild maizes and the metabolic profiling of urine and plasma samples after regular consumption of cocoa.

Regarding the matrices and analytes studied, they cover a very broad spectrum including the study of metabolites in transgenic foods, mate extracts, beers and hop extracts, as well as the determination of phenolic compounds in wheat, flavonoids in honey and licorice, glycosides in wine, volatiles in roasted coffee and wine, etc. Also, new food safety applications are shown including the analysis of trace levels of sulfonamides in honey, fluoroquinolone antibiotics in milk, pesticides in fruits and olive oils, and aromatic amines in food products and composite food packaging bags.

As editor of this special issue devoted to "Food Analysis", I would like to thank all the authors for their suitable contributions and all the reviewers for the time they devoted to the evaluation of the papers. Also, I would like to thank Dr. Salvatore Fanali for his continuous help and support, and to those of Journal of Chromatography A team whose efforts have contributed to the preparation of this special issue. 